\title{
Téoros
}

Revue de recherche en tourisme

\section{Les sociétés locales face au tourisme nudiste}

Résultats d'une enquête qualitative sur la côte pacifique du

Mexique

\section{Juan Carlos Monterrubio et Emmanuel Jaurand}

Volume 28, numéro 2, 2009

URI : https://id.erudit.org/iderudit/1024811ar

DOI : https://doi.org/10.7202/1024811ar

Aller au sommaire du numéro

Éditeur(s)

Université du Québec à Montréal

ISSN

0712-8657 (imprimé)

1923-2705 (numérique)

Découvrir la revue

Citer cet article

Monterrubio, J. C. \& Jaurand, E. (2009). Les sociétés locales face au tourisme nudiste : résultats d'une enquête qualitative sur la côte pacifique du Mexique. Téoros, 28(2), 83-92. https://doi.org/10.7202/1024811ar
Résumé de l'article

Cet article cherche à explorer les attitudes de la population résidente par rapport à la nudité publique pratiquée par des touristes et plus généralement par rapport au corps dénudé. Fondée sur des entrevues approfondies, l'étude a pour cadre une plage nudiste tolérée de la côte pacifique mexicaine. Les résultats montrent que même si la nudité collective n'est pas conforme aux valeurs culturelles et sociales de la communauté d'accueil, l'impact économique de ce tourisme joue un rôle important dans la perception positive que les habitants ont des touristes nudistes. Par conséquent, la pratique nudiste est tolérée dans la mesure où elle reste limitée aux extrémités de la plage, zones habituellement fréquentées par les nudistes. 


\title{
Les sociétés locales face au tourisme nudiste Résultats d'une enquête qualitative sur la côte pacifique du Mexique
}

\author{
Juan Carlos MONTERRUBIO \\ Centro Universitario UAEM Texcoco \\ Universidad Autónoma del Estado de México \\ carlos.monterrubio@ymail.com \\ Emmanuel JAURAND \\ Département de géographie \\ Université de Paris 12 \\ jaurand@univ-paris12.fr
}

RÉSUMÉ: Cet article cherche à explorer les attitudes de la population résidente par rapport à la nudité publique pratiquée par des touristes et plus généralement par rapport au corps dénudé. Fondée sur des entrevues approfondies, l'étude a pour cadre une plage nudiste tolérée de la côte pacifique mexicaine. Les résultats montrent que même si la nudité collective n'est pas conforme aux valeurs culturelles et sociales de la communauté d'accueil, l'impact économique de ce tourisme joue un rôle important dans la perception positive que les habitants ont des touristes nudistes. Par conséquent, la pratique nudiste est tolérée dans la mesure où elle reste limitée aux extrémités de la plage, zones habituellement fréquentées par les nudistes.

Mots-clés : Nudisme, plage, tourisme balnéaire, Mexique.

L'étude des impacts du phénomène touristique sur les sociétés et les cultures locales a été relativement négligée dans les recherches sur le tourisme par rapport aux impacts économiques (Ap, 1990). Pourtant, prendre en compte les attitudes de la population résidente par rapport aux comportements des touristes sur leurs lieux de vacances peut aider à cerner les impacts sociaux du tourisme (Getz, 1994), et améliorer la qualité de vie des communautés d'accueil (Crouch et Ritchie, 1999). En outre, identifier les attitudes de la population résidente est important pour connaître son soutien à l'activité touristique, envisager les nouvelles opportunités de développement et apporter des réponses aux problèmes posés par le tourisme (Getz, 1994; Williams et Lawson, 2001). Par la connaissance des réactions des populations locales, des mécanismes peuvent être mis en place pour diminuer d'éventuels conflits avec les touristes (Lankford et Howard, 1994), et, au-delà, contribuer à un développement touristique durable (Zhang et al., 2006). L'importance de ces études pour l'aide à la décision en matière de développement touristique local a ainsi été soulignée (Brunt et Courtney, 1999). Les enjeux sont d'autant plus forts que sont mises en contact par le tourisme international des populations mobiles originaires de pays du Nord et des populations résidentes de pays du Sud, caractérisées par des niveaux de revenus, des représentations culturelles et des pratiques sociales différentes. Produit et vecteur de la mondialisation, le tourisme international à destination des pays du Sud détermine dans les sociétés réceptrices un mélange d'attrait et de répulsion largement fondé sur des enjeux identitaires, notamment la question de la reconnaissance de l'autre (Amirou, 2005).

Notre article s'inscrit dans le champ des études touristiques et est le résultat d'un travail bidisciplinaire puisque les auteurs sont respectivement sociologue et géographe. Plus précisément, à partir de l'enquête ethnographique de terrain, nous analysons les réactions d'une communauté d'accueil par rapport à la pratique nudiste développée par des touristes, majoritairement étrangers, sur la principale plage nudiste de la côte pacifique mexicaine (Zipolite). Ce faisant, nous cherchons à accroître les connaissances sur les impacts locaux du tourisme balnéaire et de la pratique nudiste sur les sociétés d'accueil à travers une contribution ponctuelle, centrée sur les résultats d'une enquête de terrain.

La question du nudisme est pertinente pour apprécier ces impacts dans la mesure où cette pratique corporelle de nature originaire du Nord de l'Europe, où elle constitue un phénomène social enraciné, voire banalisé, en liaison avec la morale corporelle post-protestante (Macnagthen et Urry, 2001), s'est diffusée vers le reste de l'Europe et une partie du monde par 
le biais des migrations de peuplement et du tourisme international. Si dans des pays comme la Croatie, l'Espagne ou le Brésil la réception a été positive, avec même une intégration de la pratique par des nationaux, au Maghreb ou en Inde, la greffe a échoué compte tenu des réticences et obstacles des sociétés d'accueil (Jaurand, 2006). La diffusion du nudisme participe d'un transfert des modes de vie européens opéré par le tourisme international (Jacobsen, 2003; Jaurand, 2008). Il nous faudra ainsi replacer notre étude dans le contexte mexicain et, plus largement, dans celui de l'espace touristique d'Amérique centrale, sous la dépendance des foyers émetteurs nord-américain et européen. Choisir pour objet d'étude une plage (nudiste) n'est pas anecdotique par rapport à cette large perspective : Cantu (2002) souligne l'importance des images de plages dans l'attraction que le Mexique exerce sur les touristes d'Amérique du Nord.

\section{Nudisme, tourisme et attitudes des sociétés d'accueil}

\section{Le nudisme et le tourisme}

Le nudisme comme pratique de loisir et de tourisme a été pratiquement ignoré dans les recherches ayant trait au tourisme. Plus largement, les études sur le corps ont été assez limitées jusqu'à récemment dans le champ des sciences sociales (Synnott, 1993) et en particulier dans celui du tourisme, longtemps marqué par la dichotomie hiérarchique entre l'esprit et le corps (Johnston, 2001). Alors que presque huit décennies ont passé depuis les premiers écrits à caractère non médical sur le nudisme (Merrill et Merrill, 1931), la littérature universitaire sur le sujet est fort restreinte. Pourtant, la pertinence du nudisme comme sujet d'étude a été attestée d'un point de vue sociologique, historique et géographique (Douglas et al., 1977; Kaufmann, 1998; Baubérot, 2004; Barthe-Deloizy, 2003; Jaurand et de Luze, 2004). L'importance sociale des études sur le corps a aussi été reconnue dans la mesure où la nudité est indissociablement liée à l'identité anthropologique, sociale et religieuse des êtres humains (Perniola, 1989). Le nudisme constitue une pratique corporelle inscrite dans un espace à forte dimension naturelle, rompant avec les normes vestimentaires de la vie quotidienne, en même temps qu'il produit l'image d'une nature socialisée, vue comme fondamentalement bonne (Macnagthen et Urry, 2001).

Alors que la nudité publique existe depuis l'Antiquité, comme dans la civilisation grecque (Clarke, 1982), ce n'est que dans les années 1920 que le nudisme social, considéré comme un mode de vie alternatif, a commencé à se populariser dans certains pays européens, au premier rang desquels l'Allemagne. À cette époque, le nudisme y était une pratique de loisir enracinée culturellement, et concernait des couples mariés, des familles et des jeunes des deux sexes (Cluet, 1999). Malgré son existence dans l'espace public, le nudisme a jusqu'à nos jours été l'objet d'une réprobation sociale assez commune : «Nudity is a negative state, a privation, loss, dispossession... [Nudity means] finding oneself in a degraded and shamed position, typical... of those who are demented, cursed or profaned[.]» (Perniola, 1989 : 237) Une telle stigmatisation, largement répandue en dehors du berceau historique du nudisme qu'est l'Europe du Nord, explique le caractère généralement très minoritaire de la pratique à l'échelle des autres pays et pour les nudistes, et l'impératif d'une mise à distance du reste de la société (Jaurand, 2008).

La plupart des travaux sur le nudisme concernent les clubs et associations (Merrill et Merrill, 1931), et la façon dont les nudistes considèrent la nudité par comparaison avec le reste de la société dans une perspective morale (Weinberg, 1970). De plus, les recherches sur le nudisme se sont concentrées sur les sentiments, la communication et le comportement des nudistes sur certaines plages (Douglas et al., 1977). Les rares études qui ont porté sur les relations entre le tourisme et la nudité publique traitent de la diffusion spatiale de la pratique nudiste sur les plages publiques en rapport avec les flux touristiques (Jaurand, 2006).

\section{Le nudisme et les plages}

Les plages, en plus d'être un lieu de détente, voire de spiritualité (Lencek et Bosker, 1999), ont été depuis la fin du XIX siècle l'espace privilégié du développement de la nudité publique. Alors que la plupart des plages ont d'abord été le siège d'un nudisme sauvage (illégal), certaines ont fait l'objet d'une autorisation administrative, principalement en Europe, en Amérique du Nord, en Australie et en Nouvelle-Zélande, dans un but touristique (Jaurand, 2008). L'existence d'attitudes plus conciliantes de la part de certaines communautés et autorités locales a aussi permis le développement de plages où le nudisme est simplement toléré : ces plages où les nudistes se mêlent aux autres sont qualifiées de «clothing-optional» aux États-Unis. PrestonWhyte (2004: 355) souligne que «many such beaches have tacit recognition from local authorities and some have even progressed to the next stage of acquiring legal status». Les plages où se pratiquent le nudisme montrent ainsi une grande variété de statuts juridiques et de situations, allant de l'interdiction à l'autorisation, en passant par la tolérance de fait (Barthe, 2001), ce dernier cas caractérisant la plage étudiée ici.

La popularité croissante des plages nudistes depuis les années 1960 est attribuable à plusieurs raisons. Dans le cadre du mouvement naturiste, la plage nudiste constitue le lieu idéal de communion entre le corps humain et les éléments naturels (mer, soleil, sable, air) (Barthe-Deloizy, 2003). Bell et Holliday (2001) insistent particulièrement sur l'importance du contact de la totalité du corps avec les rayons solaires comme source de plaisir et de bien-être : ceci montre une évolution par rapport à l'«héliose» des premiers naturistes, motivée par des raisons médicales. De surcroît, la pratique du nudisme de plage est étroitement liée à une expérience individuelle et familiale de relaxation, de recréation et de socialisation entre personnes de mêmes affinités (Douglas et al., 1977). PrestonWhyte (2004: 355) souligne que «nudist beaches are spaces of relaxation where the atmosphere of liminality allows... to relax and release the tensions of life ». On retrouve ici un avatar de la fonction fondamentale du tourisme, la recréation de l'individu, fondé sur la rupture avec les pratiques attachées à l'univers du quotidien (Stock, 2003). La plage est également associée à la santé, à l'aventure, à la séduction et à la possibilité d'interactions sexuelles (Rojek, 1993 : 189). En particulier, une partie des plages nudistes publiques offrent certaines opportunités sexuelles pour des nudistes peu soucieux de l'éthique 
naturiste, depuis le voyeurisme et l'exhibitionnisme jusqu'au rapport sexuel lui-même (Douglas et al., 1977). Certaines plages nudistes peuvent aussi être considérées comme des échappatoires à la norme ou à l'exclusion sociale et contribuer à valider l'identité sexuelle de certains individus, en particulier les homosexuels masculins (Jaurand, 2005).

Bien que certaines plages aient obtenu une tolérance, voire une officialisation de la pratique nudiste, la nudité publique peut conduire à un choc culturel avec les sociétés d'accueil, au moins avec celles qui ont conservé des codes moraux traditionnels. Néanmoins, compte tenu de l'importance économique locale du tourisme, il peut exister une certaine tolérance de la part des habitants envers le touriste nudiste. L'éventuel rejet ou l'éventuelle désapprobation de la nudité peuvent être liés aux valeurs culturelles, sociales et religieuses des résidents aussi bien qu'au comportement des nudistes et à la nature des interactions entre les deux groupes. Il existe aussi une grande variété dans la perception du corps nu dans l'espace public: "the creation and learning of the body as a social phenomenon varies from culture to culture [...] The one word, body, may therefore signify very different realities and perceptions of reality[.]» (Synnott, $1993: 4,7$ ) On ajoutera dans ce sens qu'au sein d'un même pays les sociétés locales réagissent de façon très différente au nudisme : en France, les littoraux les plus récemment investis par le tourisme ont été les espaces d'accueil privilégiés de la pratique nudiste (Languedoc, côtes varoise, landaise et girondine), à l'opposé des littoraux de tourisme ancien et huppé comme ceux des Alpes-Maritimes ou du Pays Basque (Jaurand et de Luze, 2004).

L'acceptation du tourisme nudiste peut être fondée sur son intérêt économique ou sur l'attitude libérale et les valeurs spécifiques des sociétés d'accueil. En parallèle, d'autres paramètres entrent en jeu, notamment la perception par les sociétés d'accueil, en fonction de leurs traits culturels et de leur mentalité, du comportement des touristes nudistes. D'un point de vue social, plus grande est la différence entre les résidents et les touristes, plus important est l'impact local du tourisme (Spanou, 2007). Les impacts socioculturels du tourisme ne dépendent pas seulement du nombre de touristes, mais aussi des caractéristiques des touristes (nudistes) et de leur comportement particulier sur le lieu de destination (nudité publique) (Brunt et Courtney, 1999; Smith, 1989). Sans aucun doute, parce que le nudisme peut être considéré comme un comportement aberrant, voire un péché (Preston-Whyte, 2004 : 355), la nudité publique constitue un indicateur intéressant pour interroger les sociétés réceptrices du tourisme.

\section{Les réactions des sociétés au nudisme}

Dans l'espace public, c'est sur les plages que le nudisme s'est le plus répandu, avec des autorisations motivées par un souci de développement touristique : c'est en tant que segment touristique que le nudisme a été partiellement intégré par les sociétés (Jaurand,2006). Même si divers arguments en faveur du nudisme ont été avancés, en particulier ses effets bénéfiques sur la santé mentale et physique des individus et des groupes (Bacher, 1996), les réactions sociales au nudisme restent souvent marquées d'une certaine hostilité. De telles réactions existent jusque dans les pays où le nudisme social est le plus banalisé (Daley, 2005;
Jaurand, 2007). Ainsi, en Allemagne après la chute du rideau de fer, bien des touristes de l'ouest se rendant sur les plages de l'est ont été choqués par la prolifération du nudisme en dehors des secteurs homologués FKK (Freikörperkultur, la libre culture du corps, du nom de la fédération naturiste allemande, la première du monde fondée en 1918).

Parce que le corps est chargé de significations sociales et culturelles, qu'il engage la distinction entre sphère privée et sphère publique et qu'il est associé à la sexualité (Synnott, 1993), certaines réactions négatives au nudisme peuvent s'expliquer par des raisons morales ou religieuses. Certaines personnes considèrent en effet la nudité publique comme bizarre, détestable, incompréhensible et immorale (Douglas et al., $1977: 28$ ), et les nudistes comme des pervers sexuels (Daley, 2005 : 501). Booth (1997 : 171) affirme que «[m] oralists called [nudity] depraved and corrupt behaviour: bathing signified absence of restraint self-control over bodily desires, and it simulated desire for flesh, aroused erotic thoughts and encouraged sexual crimes ». Une telle perception de la nudité publique, systématiquement associée à la sexualité, répandue aussi bien dans les pays de religion catholique qu'islamique, a été l'objet d'une dénonciation de la part du mouvement naturiste, prônant une nudité mixte et chaste dans les centres.

Certains lieux nudistes ouverts, en particulier les plages du nudisme sauvage, sont l'objet d'une réprobation publique, voire d'une répression de la part des autorités. Preston-Whyte (2004 : 355) avance une raison à une telle condamnation : "public nudity commonly carries with it a level of social opprobrium, largely shaped by religious dogma, which tends to be articulated through expressions of disgust, disapproval, and suspicion.» La répression par la loi et les forces de l'ordre résulterait ainsi de considérations morales qui ont acquis la force de l'évidence dans la plupart des sociétés.

Même s'il existe une désapprobation presque générale du nudisme, les formes et les degrés de la moralité varient fortement d'une culture à une autre (Douglas et al., 1977). D'un point de vue moral, la conception du corps dépend autant des considérations personnelles que de l'influence culturelle (Synnott, 1993). Elle peut aussi varier en fonction du contexte du développement du nudisme (par exemple, la présence de centres naturistes intégrés au développement du tourisme balnéaire, comme en Croatie, en Espagne, au Portugal, mais aussi à Saint-Martin, en Jamaïque et à Cuba) et aussi du profil des nudistes. Jusqu'à présent il n'y a pas eu d'études sur l'influence des conceptions morales des sociétés d'accueil sur les réactions au nudisme.

\section{Les attitudes de la population résidente par rapport au tourisme}

Dans une perspective socioculturelle, les études sur les attitudes des sociétés d'accueil par rapport au tourisme ont gagné en reconnaissance ces trois dernières décennies. Leur importance théorique et pratique a été vue comme un élément décisif de l'évaluation des impacts, positifs ou négatifs, sur les sociétés et les espaces touchés par la mise en tourisme (Getz, 1994).

L'approche des attitudes envers le tourisme a principalement pris en compte des critères sociodémographiques et économiques des populations concernées : le genre, l'âge, le lieu 
de naissance et le temps de résidence sur le lieu touristique ont été retenus comme des critères intéressants pour définir les attitudes par rapport au tourisme (Monterrubio, 2008). Plus particulièrement, la dépendance économique des communautés par rapport au tourisme a été identifiée comme un critère décisif pour le soutien au développement touristique : une relation claire, certes attendue, a été établie entre les avantages économiques réels et perçus, et les attitudes des populations résidentes par rapport à l'activité touristique (Pizam, 1978). Cependant, même dans des cas où l'impact économique est jugé positif, les attitudes des populations résultent aussi de leur environnement social, culturel et même politique.

Il est aussi attesté que le degré de développement touristique, le profil des touristes et leurs comportements sur le lieu de destination, parmi lesquels la pratique nudiste, entrent en ligne de compte dans l'attitude par rapport à cet "autre» en déplacement temporaire qu'est le touriste. Et les attitudes des communautés locales face au tourisme peuvent varier significativement en fonction des conditions sociales, économiques, culturelles, légales et politiques de la destination.

\section{Cadres de l'enquête de terrain à Zipolite Les objectifs}

La présente étude est partiellement issue d'une recherche plus large dont l'objectif est d'étudier les réactions de la population résidente par rapport au comportement des touristes. À la différence d'études précédentes qui ont porté sur le comportement des touristes fréquentant une autre plage mexicaine (Passariello, 1983), notre recherche s'intéresse aux réactions de la population locale par rapport au phénomène touristique développé sur une plage de la côte pacifique. Nous souhaitons ainsi contribuer à la connaissance de la perception qu'ont les sociétés d'accueil du tourisme et particulièrement de la pratique nudiste dans un lieu touristique. La recherche vise plus précisément à apprécier les positions de la population résidente par rapport à la présence et au comportement des touristes nudistes sur leur lieu de destination (favorable, défavorable ou autre). Il s'agit aussi, à travers les entretiens, de tenter de cerner les raisons récurrentes invoquées par la communauté d'accueil pour justifier son attitude par rapport au tourisme nudiste.

\section{Le lieu et la population de l'enquête}

L'enquête a été menée sur la plage nudiste de Zipolite (illustration $1^{1}$ ), située dans une région rurale de la côte pacifique du Mexique, dans l'État d'Oaxaca, à $540 \mathrm{~km}$ à l'est d'Acapulco et à $60 \mathrm{~km}$ à l'ouest de Puerto Escondido. Cette dernière station est une destination internationale réputée pour ses compétitions de surf. Zipolite est également facilement accessible depuis l'aéroport de Huatulco, distant de la station de moins de $50 \mathrm{~km}$, et qui permet l'arrivée d'une clientèle étrangère transitant par Mexico ou de résidents de l'agglomération capitale. Zipolite est réputée dans le pays pour être la plage publique la plus importante du Mexique en termes de fréquentation par des nudistes (Brenner et Fricke, 2007). Signe de sa notoriété, elle est répertoriée jusque dans un guide touristique généraliste comme le Guide vert Michelin (2001 : 277). Le nudisme y est développé sans autorisation officielle, simplement par une tolérance de facto. Contrairement aux pays d'Amérique du Nord et aussi à certains pays d'Amérique Centrale (République Dominicaine, Cuba, Jamaïque, Panama), le Mexique ne dispose d'aucune plage où le nudisme

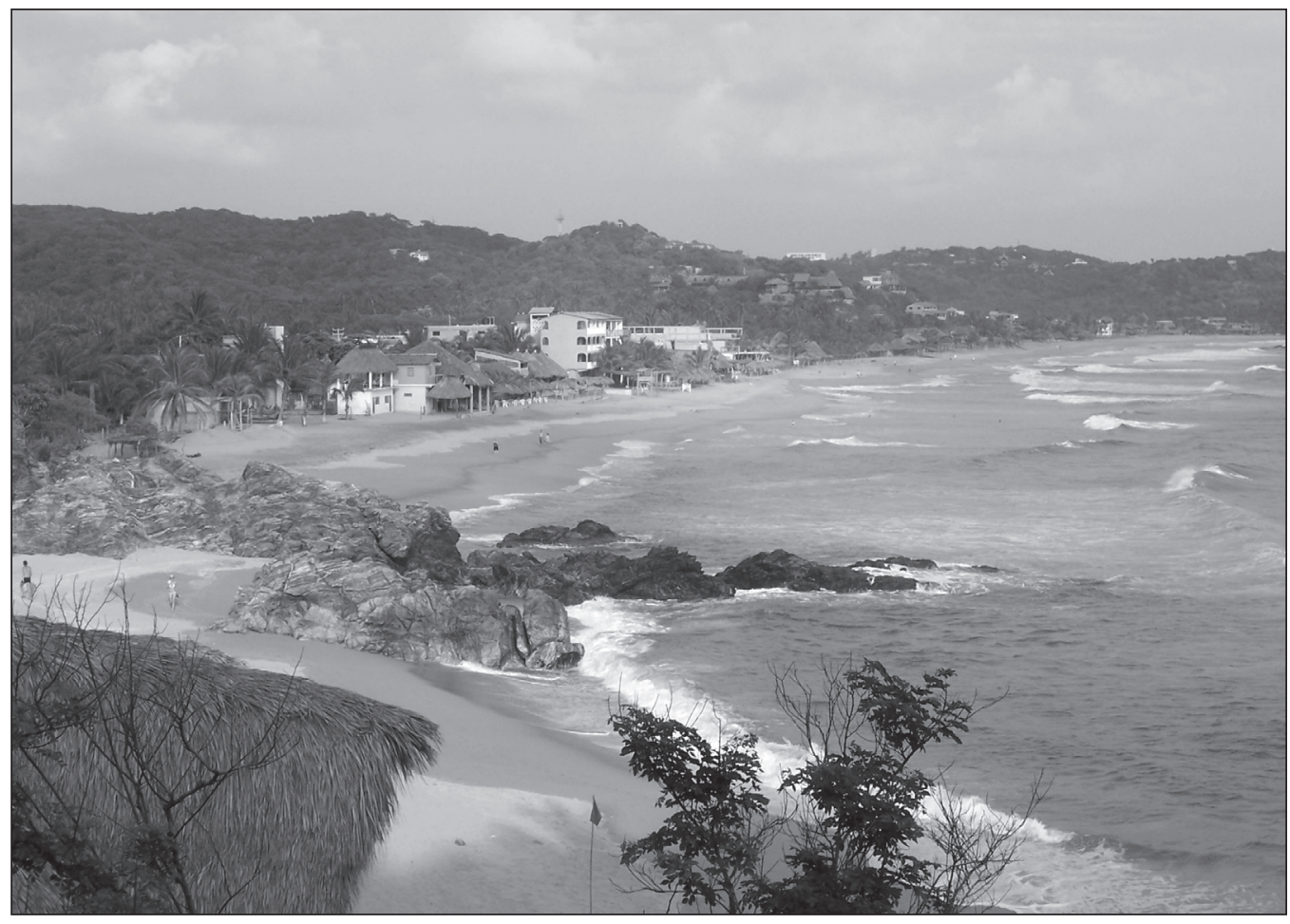

ILLUSTRATION 1 : Vue générale de la plage de Zipolite en direction de l'Est (photo : Juan Carlos Monterrubio). 
soit officiellement autorisé. La loi fédérale mexicaine ne mentionne pas explicitement les situations de nudité publique ou de nudisme, mais condamne «les comportements immoraux», ce qui suffirait à condamner une exhibition du corps nu dans l'espace public. À Zipolite, nous n'avons eu aucun écho de plaintes déposées contre les nudistes auprès des autorités. La plage elle-même relève entièrement du domaine public fédéral, mais juste au-dessus de la plage se sont multipliés depuis les années 1970 les hôtels, bars et restaurants sous forme de bâtiments le plus souvent à un ou deux étages maximum et noyés dans la verdure tropicale.

Cette plage est un terrain de recherche intéressant pour étudier la perception de la communauté d'accueil par rapport à une pratique exclusivement touristique et déjà relativement ancienne. L'attrait de cette destination repose sur sa préservation par rapport à l'urbanisation dense, la réputation de liberté de mours associée au nudisme et à la consommation de drogues dans les représentations des milieux sociaux nordaméricains marqués par la contre-culture des années 1960. Brenner et Fricke (2007 : 225) mentionnent aussi cette destination parmi les lieux du tourisme gay au Mexique, même s'il est impossible d'assimiler la localité à une station majoritairement gay comme il en existe aux États-Unis. Si la plage de Zipolite est en effet référencée dans le principal guide mondial de tourisme gay, le guide Spartacus (2007), et si le Mexique est une destination privilégiée des gays états-uniens (Cantu, 2002), les gays ne constituent qu'une partie des nudistes qui fréquentent la plage et les nudistes eux-mêmes sont minoritaires par rapport à l'ensemble des personnes qui fréquentent régulièrement la plage. On ne peut donc assimiler l'ensemble de la plage de Zipolite à une plage nudiste et a fortiori à une plage gay.

La destination étudiée n'abritait que quelques dizaines de maisons dispersées avant d'être connue comme un paradis hippie dans les années 1970 : faisant partie de la municipio (commune) de San Pedro Pochutla, elle a acquis le titre de poblacion (localité) en 1970. Il est probable que les hippies ont été les premiers promoteurs du nudisme à Zipolite, à l'instar de ce qui a été montré dans d'autres lieux comme l'île d'Ibiza (Rozenberg, 1990). À la fin des années 1970, des familles ont fait construire des maisons pour y résider et développer l'économie touristique. Aujourd'hui, Zipolite compte environ mille habitants et vit essentiellement de l'activité touristique. Tout le littoral environnant, et en particulier la localité de Puerto Angel qui jouxte Zipolite à l'est, a connu un développement touristique, qui explique la croissance démographique (Brenner et Fricke, 2007). La population locale résulte largement d'apports migratoires internes au Mexique, même si quelques Nord-Américains et Européens résident à Zipolite en permanence.

Même si le catholicisme, religion dominante à Zipolite comme dans tout le Mexique, semble assez peu pratiqué, il influence la vie de la communauté du point de vue des valeurs dominantes et du contexte moral. Cette conclusion est fondée sur la fréquente mention de la religion au cours des entretiens. Par ailleurs, il semble exister un fort sentiment d'attachement des habitants à leur communauté : cela repose sur leur participation active à la prise de décision collective et sur la fierté d'être né ou de résider à Zipolite.

\section{Les méthodes}

La majorité des études relatives aux impacts du tourisme sur les sociétés d'accueil se fonde sur des méthodes quantitatives. Cependant, d'autres études ont insisté sur la valeur des méthodes qualitatives pour interpréter les attitudes des personnes interrogées par rapport au tourisme (Brunt et Courtney, 1999; Spanou, 2007). De telles études montrent que l'utilisation d'entretiens approfondis et partiellement détachés de questionnaires systématiques ainsi que la constitution de groupes de discussion méritent d'être reconnues comme des méthodologies à part entière dans les études relatives aux impacts touristiques.

Notre étude est ainsi conduite selon une approche qualitative. Des entretiens semi-directifs ont été effectués pour connầtre les perceptions des touristes nudistes par les résidents. En effet, il est attesté que ce type d'entretien est particulièrement adapté pour connaître les représentations que les gens se font du monde et pour connaître leur propre expérience de la réalité (Kvale, 1996 : 105). La composition du questionnaire a été définie principalement à partir des travaux ethnographiques précédents et d'un examen de la littérature consacrée aux relations entre résidents et touristes. Ont été posées des questions relatives à une vision générale du tourisme nudiste, à la nature $\mathrm{du}$ contact entre personnes locales et touristes nudistes ainsi qu'au soutien à un éventuel développement supplémentaire du tourisme nudiste.

Pour le choix des personnes interrogées, nous avons combiné deux techniques. La première a consisté à s'adresser aux personnes a priori les plus informées, du fait de leur présence ancienne sur le lieu et de leur implication dans l'activité touristique (purposive sampling : Bryman, 2004). La seconde a consisté à établir des premiers contacts qui nous ont orientés vers d'autres personnes susceptibles d'apporter des réponses à notre enquête (snowball sampling). Le choix définitif des répondants repose sur différents éléments dont le plus important est la répartition spatiale par rapport au terrain d'enquête, car il est reconnu que la proximité par rapport au lieu touristique peut être un important facteur pour déterminer les attitudes des résidents (Jurowski et Gursoy, 2004). Même si l'étude ne repose pas sur un échantillon représentatif, nous avons cherché à rencontrer des répondants de profils différents. Des caractéristiques comme le rapport à la religion, le niveau d'instruction et le lieu de naissance (Zipolite ou autres) ont été prises en considération, car il a été démontré que ces critères sont les plus pertinents pour différencier les attitudes envers certaines pratiques touristiques (Madrigal, 1995). Les caractéristiques et la taille de l'échantillon sont évidemment dépendantes des techniques adoptées, et ne sont donc pas représentatives de l'ensemble de la population de Zipolite : il s'agit là d'une limite inévitable de notre enquête, liée au choix d'une méthode qualitative. D'autres travaux sociologiques utilisent les deux techniques d'échantillonnage retenues et se fondent donc sur des effectifs également limités (Valentine et Skelton, 2003).

Les entretiens ont été réalisés en espagnol au cours du dernier trimestre de l'année 2006, soit au cours de la haute saison touristique : celle-ci correspond aux premiers mois de la saison sèche tropicale, marqués par des températures pas 
trop élevées (environ $25-30{ }^{\circ} \mathrm{C}$ au maximum en journée) et un différentiel climatique maximal avec les pays développés de la zone tempérée boréale qui sont les foyers émetteurs de touristes internationaux (États-Unis, Canada, Europe). Au total, 21 personnes résidentes ont été sollicitées, mais seulement 17 entrevues approfondies ont été menées : 5 entrevues de femmes et 12 entrevues d'hommes; le déséquilibre du ratio sexuel des personnes interrogées est lié aux techniques utilisées et au caractère non représentatif de l'échantillon. Sur les 17 personnes retenues, 12 travaillent dans le secteur touristique au sens large (hôtellerie, restauration, vente ambulante sur la plage...), ce qui constitue une proportion élevée liée au choix de la technique d'échantillonnage. Les entrevues ont duré de 25 minutes à près de deux heures. Nous avons expliqué la finalité de l'enquête aux répondants et leur avons demandé l'autorisation d'enregistrer leurs propos. Les entrevues ont été conduites dans des établissements touristiques, commerciaux, à domicile ou sur la plage. Nous précisons qu'aucune des personnes interrogées ne pratique le nudisme.

\section{L'interprétation des données}

Notre étude s'est efforcée de suivre une démarche rigoureuse pour aboutir à une interprétation des données recueillies. Bien qu'il n'existe pas une méthode idéale d'analyse qualitative des données (Sandiford et Seymour, 2007 : 725), l'exploitation des entretiens a suivi la procédure indiquée par Bryman (2004). En particulier, les entretiens enregistrés ont été retranscrits tels quels et les entretiens non enregistrés ont été reconstitués sur la base des notes.

Pour le décodage de l'information, les transcriptions des entrevues ont été réorganisées sous la forme de tableaux d'analyse des données. Chaque tableau indique le numéro d'ordre, la date, l'heure et la durée de l'entretien ainsi que le nom de la personne interrogée. Le tableau se trouve divisé en quatre colonnes : 1) les thèmes et sous-thèmes; 2) la numérotation des lignes du texte retranscrit; 3 ) le texte lui-même; 4) les commentaires des chercheurs. Des commentaires plus généraux sont reportés au-dessous du tableau.

Ensuite, on a identifié des thèmes et sous-thèmes à partir du contenu des entrevues. Chacun d'entre eux correspond à une série de réactions que l'on a pu retrouver chez plusieurs répondants. Ces sous-thèmes ont été ensuite rapportés à des thèmes généraux déjà existants dans la littérature sur le sujet ou à des thèmes nouveaux apparus dans le contenu des entretiens. Les commentaires figurant dans la quatrième colonne portent principalement sur les implications théoriques, et des conclusions préliminaires sur les réactions des répondants.

\section{Bilan : une société d'accueil plutôt favorable, au prix d'une segmentation de l'espace public balnéaire}

De manière générale, les résultats de la recherche montrent que malgré une certaine variété d'opinions à l'égard du tourisme nudiste à Zipolite, une majorité se dégage en faveur de celui-ci. Bien qu'il soit parfois délicat de classer ces opinions, des attitudes rangées entre l'opposition totale, l'indifférence, et l'approbation totale ont été distinguées.

\section{L'opposition, l'indifférence et l'approbation}

L'opposition au nudisme est apparue étroitement liée à des positions conservatrices d'une partie de la communauté d'accueil, ce qui confirme pleinement les propositions exposées à ce sujet dans la littérature (Douglas et al., 1977). Des éléments comme les valeurs propres à la communauté et des normes morales ont été avancés par les répondants. Le nudisme, comme pratique de loisir, était ainsi considéré comme immoral et contraire aux valeurs locales. Un homme de 43 ans natif de Zipolite déclare par exemple : «Dans la communauté, il y a des gens qui sont contre le nudisme parce qu'ils le considèrent comme immoral... et ils pensent que c'est un mauvais exemple pour les enfants et la communauté. Ils voient les nudistes comme des pervers.»

D'autres répondants ont témoigné d'une indifférence à l'égard du tourisme nudiste. En d'autres termes, ils ont exprimé une position ni favorable ni défavorable au nudisme. Une telle indifférence était en partie attribuée par les répondants eux-mêmes à la longue période durant laquelle ils ont été au contact de visiteurs nudistes. Une femme de 53 ans dit: «En fait, je ne considère le nudisme ni comme bon ni comme mauvais. Pour moi c'est habituel parce que j'ai toujours connu le nudisme depuis que je suis née.» D'autres personnes ont pensé qu'avoir une position neutre par rapport au tourisme nudiste était une marque d'ouverture d'esprit. Pour certains, le nudisme est envisagé comme un loisir et la plage de Zipolite, comme un lieu idéal pour sa pratique. D'après un résident non natif, «[1]es gens qui ne sont pas larges d'esprit ne peuvent comprendre le nudisme. Je n'ai rien contre les nudistes. C'est ce qu'ils aiment faire et la localité leur offre cette possibilité. Il faut être ouvert pour comprendre ou au moins tolérer le nudisme». Une telle position de neutralité apparente correspond à une acceptation de facto de la pratique nudiste.

Une majorité des répondants s'est prononcée en faveur du nudisme. Ces personnes ont déclaré que les touristes nudistes, quelle que soit leur nationalité, étaient toujours les bienvenus à Zipolite. Elles allaient même plus loin en affirmant que la communauté avait besoin de développer une telle fréquentation. Un propriétaire d'hôtel âgé de 54 ans a déclaré : «Le nudisme, c'est bien. Je n'ai pas d'opinion particulière envers les touristes nudistes. Ils sont les bienvenus... En fait, il nous en faudrait plus. La localité est bien connue pour offrir la liberté aux nudistes. Si nous n'avons pas les nudistes, alors nous n'avons plus d'argent, c'est aussi simple que cela.»

Comme plusieurs intervenants l'ont souligné, la contribution économique du tourisme nudiste a été citée comme un élément important de l'acceptation des touristes nudistes. Ainsi, il peut être retenu que l'approbation du nudisme est largement redevable aux retombées financières qu'une telle pratique touristique apporte à la communauté.

En outre, d'autres répondants, non engagés dans le secteur touristique, pensent qu'il convient de protéger et de maintenir le nudisme à Zipolite, arguant qu'il est un élément original de la destination à l'échelle du Mexique. Un homme non natif a pour sa part confié : «Même si je n'ai pas grandi ici, j'ai appris à tolérer le nudisme. En fait, je pense que le nudisme devrait être conservé... Les touristes se dénudent 
seulement sur la plage. Quand ils consomment dans les bars et restaurants, même sur la plage, ils s'habillent. Ils ne sont jamais importuns de même qu'ils ne nous dérangent pas.» Certains répondants favorables au nudisme le considèrent comme un autre mode de vie ou une activité de loisir spécifique aux étrangers. Pour eux, il ne devrait pas y avoir d'opposition au tourisme nudiste, car le nudisme est «quelque chose de normal» et devrait être traité comme tel. Un résident âgé de 30 ans affirme ainsi : "Le nudisme est une réalité du monde. Les touristes nudistes n'ont pas de mauvaises intentions quand ils se dénudent sur la plage... On devrait apprendre aux enfants que le nudisme est quelque chose de normal et [qu'il] fait partie de la nature humaine. Si les enfants sont élevés ainsi, ils accepteront et respecteront le nudisme. Je pense que le nudisme est mal pour les gens qui ne sont pas tolérants envers les autres modes de vie.»

Au total, 16 répondants sur 17 se sont déclarés indifférents ou favorables au nudisme, une telle position étant le plus souvent conditionnée par un encadrement spatial de la pratique.

\section{L'extension et les limites spatiales du nudisme}

Il est important de noter que la pratique du nudisme n'est pas généralisée sur la totalité de la plage de Zipolite, dessinant un arc de cercle largement ouvert vers le sud de près de $2,5 \mathrm{~km}$ de long (illustration 2). Il apparaît même, derrière l'approbation du nudisme par plusieurs répondants, que le nudisme est accepté dans la mesure où il ne concerne que quelques parties bien définies de la plage. Ces secteurs ne sont aucunement délimités officiellement; ils l'ont été "spontanément» par les pratiques des nudistes, en fonction de leur perception des lieux et d'une logique de mise à distance.

Même si la plage de Zipolite est recensée dans certains guides comme une plage nudiste, il est important de noter que la population locale ne l'envisage pas comme exclusivement dévolue au nudisme. D'ailleurs, l'observation de la pratique montre que les non-nudistes y sont généralement très majoritaires. Selon les répondants, il y a certaines parties spécifiques réservées au nudisme. Ils divisent la plage en trois parties : la partie centrale et les deux extrémités. Alors que la partie centrale est considérée comme une "plage familiale», les extrémités ouest et est, qui représentent moins d'un sixième de la surface totale de la plage, sont réputées comme secteurs nudistes, ce qui correspond à la pratique effectivement constatée (illustration 3). Il faut signaler cependant qu'une publication ancienne (Perez, 1978) et quelques répondants indiquent qu'à l'origine seule l'extrémité orientale de la plage, bien connue sous le nom de la playa del amor (la plage de l'amour), était nudiste. Une telle répartition de la pratique nudiste aux seules extrémités d'une plage, constitue une configuration spatiale fort commune, renvoyant au modèle centre/périphérie : nous l'avions observée sur la plupart des plages de la Côte d'Azur où les nudistes s'isolaient sur une portion de plage éloignée des «textiles» et du point d'accès principal (Jaurand et de Luze, 2004).

Dans ce sens, une remarque faite fréquemment par plusieurs répondants concerne l'extension spatiale du nudisme sur la plage de Zipolite. Chez ces résidents, il y a l'idée que le nudisme s'est étendu en des endroits qui étaient autrefois «textiles». Quelques résidents ont désapprouvé une telle «invasion nudiste» parce qu'ils souhaitaient préserver des zones familiales à la fois pour les touristes «ordinaires» et les familles locales. Selon un homme de 24 ans : «Il y a certains endroits pour être nu : ... les extrémités de la plage en fait. On peut $y$ trouver beaucoup de nudistes. Mais il y a des personnes qui se dénudent sur le reste de la plage alors que beaucoup des visiteurs et aussi des habitants de Zipolite profitent de la plage sans être nus. Ainsi je ne suis pas d'accord pour qu'ils fassent du nudisme partout.» On retiendra ici que la question du lieu et de la distance permettant l'évitement de l'autre est essentielle pour accepter ou tolérer une pratique sociale différente de celle communément admise.

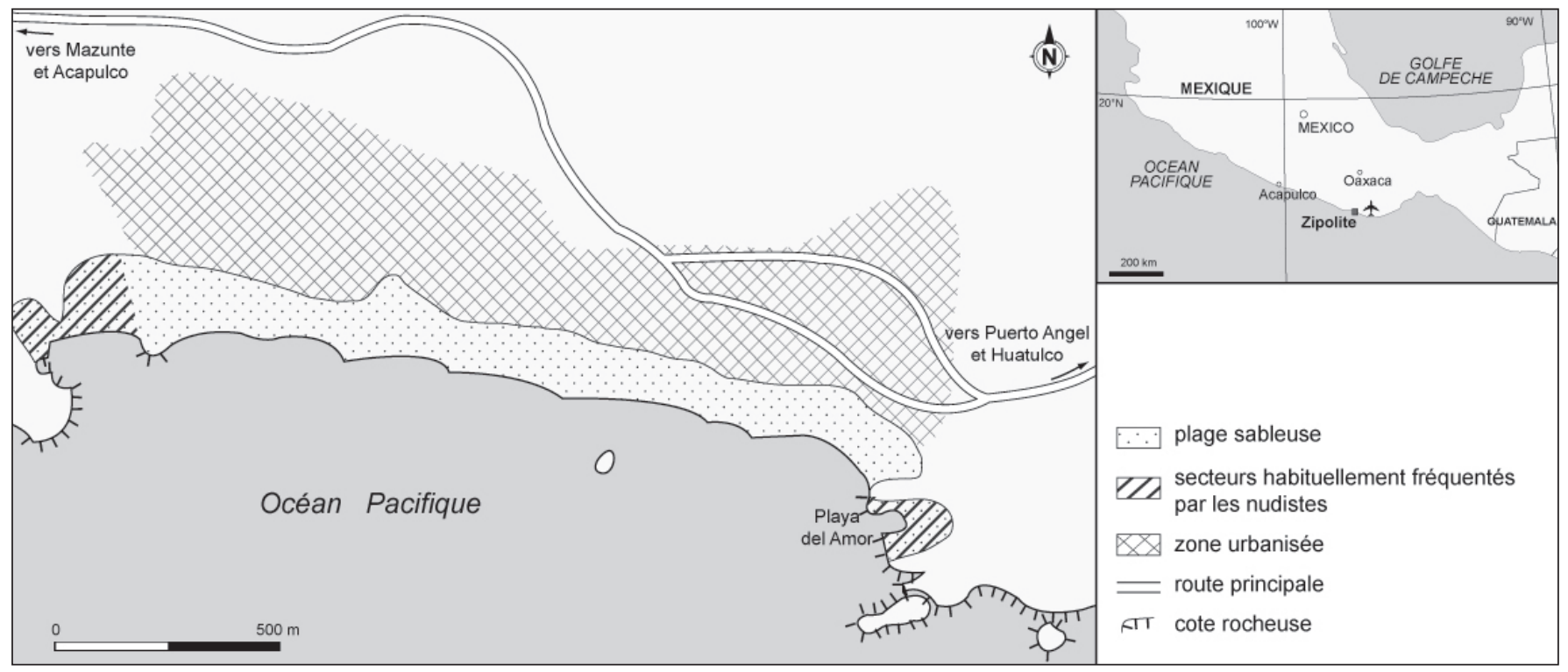

ILLUSTRATION 2 : La plage de Zipolite (source : Emmanuel Jaurand et Juan Carlos Monterrubio). 


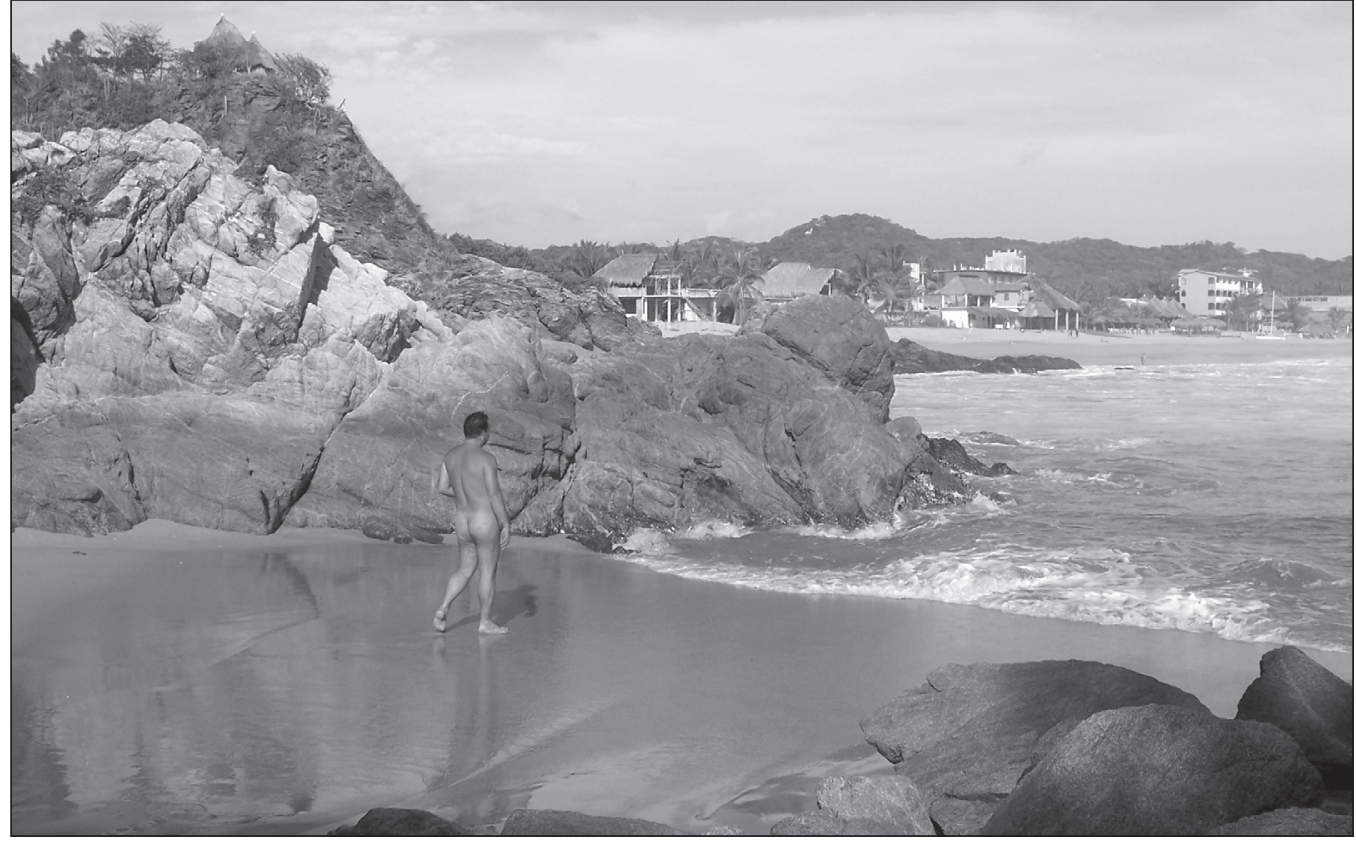

ILLUSTRATION 3 : Nudiste sur la plage de Zipolite (photo : Juan Carlos Monterrubio).

\section{La question du soutien à un développement accru du tourisme nudiste}

Après l'identification des opinions générales à propos du tourisme nudiste, nous nous sommes également intéressés à la question d'un possible accroissement de la fréquentation de la destination par les nudistes. Notre objectif était de comprendre les raisons d'un soutien des répondants à cette éventualité. Dans l'ensemble, il est apparu qu'une majorité de répondants souhaitaient maintenir la possibilité de pratique nudiste et envisageaient favorablement une augmentation du nombre de nudistes.

Pour les répondants opposés à une telle augmentation, la raison était que le tourisme nudiste s'accompagnait d'impacts gênants pour la communauté. En particulier, les répondants refusaient davantage de nudistes parce que, de leur point de vue, la nudité publique risquait de détourner un certain nombre de visiteurs non nudistes de la destination. Ils précisaient que le nudisme risquait de gêner les résidents et les autres touristes, et ainsi dissuader ces derniers de revenir à Zipolite. L'argument d'un homme de 24 ans était : «Je ne suis pas d'accord pour qu'on fasse du nudisme partout. Cela gêne certaines personnes. Certains visiteurs n'aiment pas cela du tout. Ainsi s'ils voient des gens nus tout le long de la plage, il est peu probable qu'ils y reviennent une autre fois.» On notera ici que si l'argument économique de la fréquentation de la localité par les touristes explique une position favorable au nudisme, il est aussi invoqué par d'autres répondants pour refuser son développement. Cela renvoie à une limite maximale de fréquentation acceptable, invoquée par les répondants non engagés dans le secteur touristique. On soulignera cependant que la notion de capacité de charge touristique, utilisée par l'Organisation mondiale du tourisme pour la gestion de secteurs littoraux, voire de plages, n'est pas clairement définie et est contestée par certains auteurs (Deprest, 1997).
Alors que des répondants refusent qu'il y ait davantage de nudistes, d'autres l'accepteraient sous réserve de certaines conditions. Ces conditions sont liées au confinement spatial de la pratique nudiste et au type de pratique nudiste. Les répondants suggèrent qu'ils admettraient plus de nudistes dans la mesure où ceux-ci respecteraient le secteur non nudiste de la plage et s'abstiendraient de tout comportement sexuel, conformément à l'éthique naturiste. Une femme de 50 ans originaire de Zipolite nous a ainsi déclaré : "Plus de nudistes, d'accord, mais seulement ceux qui viennent profiter de la nature et de la beauté de la plage, pas ceux qui viennent s'exhiber ou faire des choses du même genre.» Aux limites spatiales de la fréquentation nudiste s'ajoutent ainsi des limites morales sur le contenu et le sens attachés à la pratique.

\section{Conclusion}

Notre recherche a montré qu'il existait une relative diversité de réactions face au tourisme nudiste dans la communauté d'accueil de Zipolite, du rejet (rare) à l'acceptation totale. Ce résultat confirme l'idée qu'il est difficile d'obtenir un consensus général à propos de la nudité publique. Ainsi que l'a suggéré Synnott (1993 : 37), ce problème tient aux aspects culturels attachés à la construction sociale du corps nu : «At present, there is no consensus on the meaning of the [naked] body and, in a pluralistic society, no consensus can be expected. Constructions reflect the values not only of the culture, but also of the sub-culture, and of the specific individuals, and they are ever-changing. » Cependant, on soulignera la faiblesse des réactions d'hostilité, alors que le nudisme ne fait pas partie des pratiques sociales traditionnelles des pays catholiques, spécialement latino-américains.

L'existence d'une dépendance économique de la communauté d'accueil par rapport au tourisme nudiste a clairement une influence considérable sur son attitude. Cela confirme que 
l'impact économique du tourisme conduit à une perception positive de celui-ci (Williams et Lawson, 2001). Notre étude a montré que pour la majorité des répondants, les bénéfices économiques du tourisme l'emportent sur les désagréments liés au nudisme et perçus par la communauté. Alors que le nudisme lui-même n'est pas toujours pleinement accepté (le contexte moral et religieux est invoqué), davantage de tourisme nudiste serait acceptable. En termes théoriques, cela suggère que les aspects économiques du tourisme sont une priorité pour les répondants, quand bien même le comportement des touristes serait discordant avec les valeurs de la société locale.

Comme dans toute autre étude à caractère plus monographique que théorique ou général, les résultats de notre enquête sur une petite destination touristique sont largement dépendants des caractères particuliers de celle-ci et ne permettent pas de tirer des généralisations valables pour d'autres destinations. On peut tout de même relier l'attitude plutôt favorable des personnes interrogées à Zipolite au caractère récent du développement touristique et urbain de la localité. Comme il a été observé en France, première destination mondiale du tourisme nudiste (Barthe, 2001), la popularisation du nudisme, qui a accompagné les bouleversements culturels et l'évolution de l'image du corps à partir des années 1960, concerne principalement les littoraux languedocien et aquitain. L'investissement de ces espaces par les nudistes a été concomitant de la phase principale de leur mise en tourisme. À Zipolite, le nudisme est indissociable de la genèse du lieu touristique à partir des années 1970. Il a largement contribué à l'image de marque de la localité dans tout le Mexique, même si le nudisme reste une pratique minoritaire sur la plage. Les réponses des personnes interrogées, majoritairement favorables au nudisme, peuvent s'expliquer par des raisons économiques, difficiles à démêler du rôle du contexte social, culturel, voire politique, marqué par l'intégration originelle du nudisme au développement touristique de la localité.

Enfin, les méthodes particulières utilisées dans notre recherche laissent apparaître d'autres limites. Même si le choix d'approches qualitatives s'avère utile pour des enquêtes sur des thèmes «sensibles", nous avons conscience des biais inhérents à de telles méthodes. Ainsi, en l'absence d'échantillon représentatif, les résultats ne peuvent pas être généralisés à l'ensemble de la communauté d'accueil. Le recours à des méthodes quantitatives pourrait permettre de confirmer ou d'infirmer les résultats obtenus, de même que l'on pourrait mener des enquêtes analogues sur d'autres destinations nudistes dans des contextes différents. En particulier, il faudrait envisager d'étudier des cas dans lesquels le tourisme nudiste est venu comme une greffe sur un espace touristique anciennement constitué et n'a pas d'impact économique important sur la population résidente, et d'autres cas comme en Europe du Nord, dans lesquels il existe une tolérance plus générale par rapport à la nudité publique.

Note

1 On pourra trouver des photographies en couleurs de la plage de Zipolite à l'adresse suivante : http://www.puertoangel.net/zipolite/ zipolite_es.html

\section{Bibliographie}

AMIROU, Rachid (2005) «Imaginaire de la mondialisation et reconnaissance culturelle», p. 61-86, dans Rachid Amirou, Philippe Bachimon, Jean-Michel Dewailly et Jacques Malezieux (dir.), Tourisme et souci de l'autre, Paris, L'Harmattan. $362 \mathrm{p}$.

AP, John (1990) «Residents'perceptions research on the social impacts of tourism ", Annals of Tourism Research, $\mathrm{n}^{\circ} 17$ (4), p. 610-616.

BACHER, K. (1996) «205 arguments and observations in support of naturism ", The Magazine of Naturist Living, $\mathrm{n}^{\circ} 16,<$ www.naturistsociety. com>, consulté en juin 2007.

BARTHE, Francine (2001) «Géographie du naturisme. À la recherche de l'éden », Géographie et Cultures, n 37, p. 37-58.

BARTHE-DELOIZY, Francine (2003) Géographie de la nudité. Être nu quelque part, Paris, Bréal. 239 p.

BAUBÉROT, Arnaud (2004) Histoire du naturisme. Le mythe du retour à la nature, Rennes, Presses Universitaires de Rennes. 348 p.

BELL, David et Ruth HOLLIDAY (2001) «Naked as nature intented», p. 127140, dans P.M. Macnaghten et J. Urry (2001) Bodies of Nature, London, Sage Publications. 202 p.

BOOTH, Douglas (1997) «Nudes in the sand and perverts in the dunes", Journal of Australian Studies, $\mathrm{n}^{\circ}$ 53, p. 170-183.

BRENNER, Ludger et Jörn FRICKE (2007) «The evolution of backpacker destinations. The case of Zipolite, Mexico ", International Journal of Tourism Research, $\mathrm{n}^{\circ} 9$ (3), p. 217-130.

BRUNT, Paul et Paul COURTNEY (1999) «Host perceptions of sociocultural impacts», Annals of Tourism Research, $n^{\circ} 26$ (3), p. 493-515.

BRYMAN, Alan (2004) Social Research Methods, Oxford, Oxford University Press.

CANTU, Lionel (2002) «De ambiente. Queer tourism and the shifting boundaries of Mexican male sexualities ", GLQ: A Journal of Lesbian and Gay Studies, n ${ }^{\circ} 8$ (1-2), p. 139-166.

CLARKE, Magnus (1982) Nudism in Australia, Victoria, Deakin University Press. $362 \mathrm{p}$.

CLUET, Marc (1999) La «libre culture». Le mouvement nudiste en Allemagne depuis ses origines au seuil du XXe siècle jusqu'à l'arrivée de Hitler au pouvoir (1905-1933), Thèse de Doctorat d'État de l'Université de Paris IV. 1145 p.

CROUCH, Geoffrey I. et J. R. Brent RITCHIE (1999) «Tourism, competitiveness, and societal prosperity», Journal of Business Research, $\mathrm{n}^{\circ} 44$ (3), p. 137-152.

DALEY, Caroline (2005) «From bush to beach. Nudism in Australasia», Journal of Historical Geography, nº 31 (1), p. 149-167.

DEPREST, Florence (1997) Enquête sur le tourisme de masse. L'écologie face au territoire. Paris, Belin. 207 p.

DOUGLAS, Jack D.; Paul K. RASMUSSEN et Carol Ann FLANAGAN (1977) The Nude Beach, California, Sage Publications. 244 p.

GETZ, Donald (1994) «Residents' attitudes towards tourism. A longitudinal study in Spey Valley, Scotland», Tourism Management, $\mathrm{n}^{\circ} 15$ (4), p. 247 258.

Guide Vert (2001), Mexique, Guatemala, Belize, Paris, Michelin, Éditions des Voyages. $413 \mathrm{p}$.

JACOBSEN, Jens Kristian Steen (2003) «The tourist bubble and the Europeanisation of Holiday Travel ", Journal of Tourism and Cultural Change, $\mathrm{n}^{\circ} 1$ (1), p. 71-87.

JAURAND, Emmanuel (2005) «Territoires de mauvais genre? Les plages gays», Géographie et Cultures, no 54, p. 71-84.

JAURAND, Emmanuel (2006) «Le tourisme naturiste en Méditerranée. Entre interface et choc des civilisations», bulletin de l'Association des Géographes Français, n 3 , p. 331-340. 
JAURAND, Emmanuel (2007) «Les espaces du naturisme : modèle allemand et exception française? », Revue Géographique de l'Est, $\mathrm{n}^{\circ} 40$ (1), p. 23-33.

JAURAND, Emmanuel (2008) «Les plages nudistes, une exception occidentale?», Géographie et Cultures, nº 67, p. 47-64.

JAURAND, Emmanuel et Hubert DE LUZE(2004) « Ces plages ou les genres s'affichent? Les territoires du sur la Côte d'Azur», dans Christine Bard, Le Genre des Territoires: Masculin, Féminin, Neutre. Angers, Presses de l'Université d'Angers, p. 227-240.

JOHNSTON, Lynda (2001) «(Other) bodies and tourism studies», Annals of Tourism Research, $\mathrm{n}^{\circ} 28$ (1), p. 180-201.

JUROWSKI, Claudia et Dogan GURSOY (2004) «Distance effects on residents' attitudes toward tourism ", Annals of Tourism Research, n ${ }^{\circ} 31$ (2), p. 296-312.

KAUFMANN, Jean-Claude (1998) Corps de femmes, regards d'hommes. Sociologie des seins nus. Paris, Nathan, coll. «Pocket». 294 p.

KVALE, Steinar (1996) Interviews. An Introduction to Qualitative Research Interviewing, London, Sage Publications.

LANKFORD, Samuel V. et Dennis R. HOWARD (1994) «Developing a tourism impact attitude scale», Annals of Tourism Research, no 21 (1), p. 121-139.

LENCEK, Lena et Gideon BOSKER (1999) The Beach. The History of Paradise on Earth, London, Penguin Books. 310 p.

MACNAGHTEN, Phil M. et John URRY (2001) Bodies of Nature, London, Sage Publications. 202 p.

MADRIGAL, Robert (1995) «Residents' perceptions and the role of government», Annals of Tourism Research, $\mathrm{n}^{\circ} 22$ (1), p. 86-102.

MERRILL, Frances et Mason MERRILL (1931) Among the Nudists. Early Nudism, London, Lightning Source UK Ltd.

MONTERRUBIO, Juan Carlos (2008) «Residents' perception of tourism : a critical theoretical and methodological review ", CIENCIA Ergo Sum, $\mathrm{n}^{\circ} 15$ (1), p. 35-44.

PASSARIELLO, Phyllis (1983) «Never on Sunday? Mexican tourists at the beach ", Annals of Tourism Research, n 10, p. 109-122.

PÉREZ, Adriana (1978) «Puerto Angel y su playa nudista», Contenido, décembre, p. 56-58.

PERNIOLA, M. (1989) «Between clothing and nudity», dans M. Feher (édit.), Fragments for a History of the Human Body, Part two, New York, Urzone, Inc., p. 237-265.

PIZAM, Abraham (1978) «Tourism'impacts : the social costs of destination community as perceived by its residents", Journal of Travel Research, $n^{\circ} 16(4)$, p. 8-12.

PRESTON-WHYTE, Robert (2004) «The beach as a liminal space», dans A. Lew, M. Hall et A. Williams, A Companion to Tourism, Oxford, Blackwell, p. 349-359.

ROJEK, Chris (1993) Ways of escape. Modern transformations in leisure and travel, London, Macmillan.

ROSENBERG, Danielle (1990) Tourisme et utopie aux Baléares. Ibiza, une ille pour une autre vie, Paris, L'Harmattan. 200 p.

SANDIFORD, Peter John et Diane SEYMOUR (2007) «A discussion of qualitative data analysis in hospitality research with examples from an ethnography of English public houses ", Hospitality Management, $\mathrm{n}^{\circ} 26$ (3), p. 724-742.

SMITH, Valene (1989) Hosts and Guests: The anthropology of Tourism, $2^{\mathrm{e}}$ éd., Philadelphia, University of Pennsylvania Press.

SPANOU, Elena (2007) «The impact of tourism on the sociocultural structure of Cyprus », Tourismos: An International Multidisciplinary Journal of Tourism, $\mathrm{n}^{\circ} 2$ (1), p. 145-162.
Spartacus International Gay Guide (2007) Berlin, Bruno Gmünder Verlag, $35^{e}$ éd. $1242 \mathrm{p}$.

STOCK, Mathis (2003) Le tourisme. Acteurs, lieux et enjeux, Paris, Belin. $299 \mathrm{p}$.

SYNNOTT, Anthony (1993) The body social: Symbolism, self, and society, London, Routledge.

VALENTINE, Gill et Tracey SKELTON (2003) «Finding oneself, losing oneself : the lesbian and gay «scene» as a paradoxical space», International Journal of Urban and Regional Research, $\mathrm{n}^{\circ} 27$ (4), p. 849-866.

WEINBERG, M. (1970) «Sexual modesty, social meanings, and the nudist camp ", dans J. Douglas, Observations of deviance, Washington: University Press of America, p. 28-34.

WILLIAMS, John et Rob LAWSON (2001) "Community issues and resident opinions of tourism", Annals of Tourism Research, nº 28 (2), p. 269-290.

ZHANG, Jiaying; Robert J. INBAKARAN et Mervyn S. JACKSON (2006) "Understanding community attitudes towards tourism and host-guest interaction in the urban-rural border region ", Tourism Geographies, $\mathrm{n}^{\circ} 8$ (2), p. 182-204. 